\title{
SÍNDROME INFLAMATORIO MULTISISTÉMICO ASOCIADO A COVID-19 EN NIÑOS: SERIE DE CASOS EN UN HOSPITAL PEDIÁTRICO DE PERÚ
}

\author{
Liz E. De Coll-Vela (1D 1,2,a , Mariela K. Zamudio-Aquise (iD) ${ }^{1,3, b}$, Héctor Nuñez-Paucar (iD),2,b, \\ Raúl R. Bernal-Mancilla (iD ${ }^{1,2, a}$, Sandra C. Schult-Montoya (iD ${ }^{1,2, a}$, Manuel Ccorahua-De La \\ Paz (iD) 1,b Cynthia L. Huby-Muñoz (iD) 1,4,a , Carlos F. Castillo-Torres (iD) ${ }^{1,4, a}$, Jorge L. \\ Candela -Herrera (iD) ${ }^{1,3, c}$, Franklin Aranda-Paniora (D) ${ }^{1,2, a}$, Raúl A. Rojas-Galarza (iD),d \\ ${ }^{1}$ Instituto Nacional de Salud del Niño Breña, Lima, Perú. \\ ${ }^{2}$ Universidad Nacional Mayor de San Marcos, Lima, Perú. \\ ${ }^{3}$ Universidad Peruana Cayetano Heredia, Lima, Perú. \\ ${ }^{4}$ Universidad de San Martín de Porres, Lima, Perú. \\ ${ }^{\mathrm{a}}$ Médico Pediatra; ${ }^{\mathrm{b}}$ Médico Pediatra Neumólogo; ${ }^{\mathrm{c}}$ Médico Pediatra Infectólogo; ${ }^{\mathrm{d}}$ Médico Pediatra Gastroenterólogo.
}

\section{RESUMEN}

La pandemia de COVID-19 ha traído una nueva afección grave e inusual denominada Síndrome Inflamatorio Multisistémico en niños, de la cual aún hay mucho por conocer. Presentamos una serie de 8 casos atendidos en el Instituto Nacional de Salud del Niño, Lima Perú. La edad media fue 5,1 años. La presentación clínica incluyó fiebre, problemas gastrointestinales agudos, afectación ocular y mucocutánea. Cuatro cumplieron criterios para Enfermedad de Kawasaki clásica. Todos tuvieron serología positiva para SARS-CoV-2, hemograma patológico, marcadores inflamatorios elevados y pruebas de coagulación alteradas. Cinco casos presentaron hipertransaminasemia y tres retención nitrogenada. Cuatro casos cumplieron criterios para Síndrome de Activación Macrófagica. Todos recibieron inmunoglobulina intravenosa, corticoides y ácido acetil salicílico. Ninguno desarrolló aneurismas coronarios. Solo uno presentó miocarditis, shock y requirió ingreso a Unidad de Cuidados Intensivos. La mayoría evolucionaron favorablemente. En todo niño con fiebre, síntomas gastrointestinales y dermatológicos; asociado a exposición al SARS-CoV-2, debe investigarse compromiso multisistémico.

Palabras clave: Enfermedad por Coronavirus 2019-nCoV; Enfermedad de Kawasaki; Niño; Inmunoglobulinas Intravenosas (Fuente: DeCS BIREME).

\section{COVID-19-ASSOCIATED MULTISYSTEM INFLAMMATORY SYNDROME IN CHILDREN: CASE SERIES AT A PEDIATRIC HOSPITAL IN PERU}

\begin{abstract}
During the COVID-19 pandemic, a new, severe and unusual condition called Multisystem Inflammatory Syndrome in children emerged, from which there is still much to learn. We report 8 children admitted to Instituto Nacional de Salud del Niño, in Lima, Perú. Their mean age was 5,1 years. Their clinical presentation included fever, acute gastrointestinal symptoms, ocular and mucocutaneous involvement. Four patients met criteria for classic Kawasaki Disease. All the patients had positive serology for SARS-CoV-2, abnormal complete blood counts and coagulation tests, and elevated inflammatory markers. Five had elevated liver enzymes and three had kidney involvement. Four patients met criteria for Macrophage Activation Syndrome. All of them received intravenous immune globulin, corticosteroids and aspirin. No coronary aneurysms were identified. Only one developed miocarditis, shock and was admitted to the Pediatric Intensive Care Unit. Most patients recovered successfully. Every child with fever, gastrointestinal and dermatological symptoms, associated with prior exposure to SARS-CoV-2, should be investigated for multi-systemic compromise.
\end{abstract}

Keywords: 2019 Novel Coronavirus Disease; Kawasaki Disease; Child; Intravenous Immune globulins. (Source: MeSH NLM).

\section{INTRODUCCIÓN}

Correspondencia: Liz Eugenia De Coll Vela; lizeugenia18@gmail.com.

Las manifestaciones del SARS-CoV-2 en niños son generalmente menos frecuentes y graves ${ }^{(1)}$. Sin embargo, en abril del 2020 surgieron informes en Estados Unidos y Reino Unido, de un cuadro agudo inflamatorio multisistémico en niños que conducía a falla multiorgánica y shock ${ }^{(2,3)}$. Este cuadro presentaba similitudes con la Enfermedad de Kawasaki (EK) y el Síndrome de Shock Tóxico (SST), con síntomas gastrointestinales, compromiso cardiaco y marcadores inflamatorios elevados consis- 
tentes con COVID-19 grave; esta condición ha sido denominada Síndrome Inflamatorio Multisistémico en niños (MIS-C) ${ }^{(4-6)}$.

Riphagen et al. reportaron en Londres la primera serie; ocho niños con cuadro similar a EK, dos positivos a SARSCoV-2. Todos desarrollaron shock y miocarditis, uno desarrolló aneurisma gigante coronario ${ }^{(3)}$. Posteriormente Verdoni et al. describieron diez casos en Bérgamo, Italia, cinco cumplían criterios para EK clásica, $80 \%$ presentó serología para SARSCoV-2, 50\% presentó shock y dos desarrollaron aneurismas coronarios. Al compararlos con casos de EK prepandemia, encontraron que tenían edad media mayor (7,5 vs 3 años), mayor compromiso cardiaco, shock y Síndrome de Activación Macrófagica (SAM) ${ }^{(7)}$. A estos estudios les siguieron Belhadjer et al. en Francia y Suiza con 35 casos, Whittaker et al. en Reino Unido con 58 casos, Toubiana et al. en Francia con 21 casos, y Cheung et al. en Nueva York con 17 casos $^{(8-11)}$. Como la pandemia llegó más tarde a América Latina, aún no se han publicado datos respecto a este síndrome en la región.

La Organización Mundial de la Salud (OMS) define como caso a menores de 19 años con fiebre $\geq 3$ días, marcadores inflamatorios elevados, evidencia de infección por SARS-CoV-2 y ninguna otra etiología microbiana; con afectación de al menos 2 sistemas: dermatológico (rash, conjuntivitis no exudativa, inflamación mucocutánea), hemodinámico (hipotensión, shock), cardiaco (disfunción de miocardio, pericardio, valvular o coronario), hematológico (coagulopatía), digestivo (vómitos, diarrea, dolor abdominal) ${ }^{(5)}$. El Centro para el Control y la Prevención de Enfermedades (CDC) también ha planteado una definición de caso con criterios similares a los de la OMS, la definición del CDC difiere en el grupo de edad (menores de 21 años), duración de la fiebre ( $\geq 1$ día) y la condición de severidad del paciente para requerir hospitalización ${ }^{(6)}$.

El objetivo de este reporte es describir las características clínicas de una serie de ocho casos de niños peruanos con Síndrome Inflamatorio Multisistémico asociado a COVID-19.

\section{REPORTE DE CASOS}

Reportamos ocho pacientes con MIS-C que ingresaron al Instituto Nacional de Salud del Niño entre el 25 de mayo y

Tabla 1. Descripción de características clínicas, tratamiento y evolución de pacientes con MIS-C asociado a COVID-19

\begin{tabular}{|c|c|c|c|c|c|c|c|c|}
\hline Característica & Caso 1 & Caso 2 & Caso 3 & Caso 4 & Caso 5 & Caso 6 & Caso 7 & Caso 8 \\
\hline Edad (años) & 6 & 7 & 0,25 & 5 & 7 & 2 & 9 & 5 \\
\hline Género & Masculino & Femenino & Femenino & Masculino & Masculino & Femenino & Masculino & Masculino \\
\hline Comorbilidades & No & No & No & No & Obesidad & No & No & No \\
\hline $\begin{array}{l}\text { Contacto con caso } \\
\text { confirmado }\end{array}$ & Padres, abuela & $\begin{array}{l}\text { Madre, tía } \\
\text { materna }\end{array}$ & $\begin{array}{l}\text { Madre, } \\
\text { abuela }\end{array}$ & Abuela, papá & No & Madre & Padres & Madre \\
\hline TE (días) & 4 & 4 & 7 & 5 & 7 & 4 & 4 & 4 \\
\hline Síntomas gastrointestinales & $\begin{array}{l}\text { Vómitos, } \\
\text { diarrea }\end{array}$ & $\begin{array}{l}\text { Dolor } \\
\text { abdominal, } \\
\text { vómitos, } \\
\text { diarrea }\end{array}$ & Diarrea & $\begin{array}{l}\text { Vómitos, } \\
\text { diarrea }\end{array}$ & $\begin{array}{c}\text { Dolor } \\
\text { abdominal, } \\
\text { vómitos, diarrea }\end{array}$ & $\begin{array}{l}\text { Vómitos, } \\
\text { dolor } \\
\text { abdominal }\end{array}$ & $\begin{array}{l}\text { Dolor abdominal, } \\
\text { vómitos, diarrea }\end{array}$ & $\begin{array}{c}\text { Dolor } \\
\text { abdominal }\end{array}$ \\
\hline Enfermedad de Kawasaki & Completo & Completo & Incompleto & Completo & No & Incompleto & Incompleto & Completo \\
\hline $\begin{array}{l}\text { Fiebre } \geq 4-5 \text { días } \\
\text { (al momento de } \\
\text { diagnóstico) }\end{array}$ & Sí (4 días) & Sí (6 días) & Sí (7 días) & Sí (5 días) & No (3 días) & Sí (5 días) & Sí (4 días) & Sí (4 días) \\
\hline Inyección conjuntival & Sí & Sí & Sí & Sí & Sí & Sí & Sí & Sí \\
\hline $\begin{array}{l}\text { Cambios en labios y/o } \\
\text { cavidad oral }\end{array}$ & $\mathrm{Si}$ & Sí & No & Sí & No & No & Sí & Sí \\
\hline $\begin{array}{l}\text { Cambios en } \\
\text { extremidades periféricas }\end{array}$ & Sí & Sí & No & Sí & No & Sí & No & Sí \\
\hline Exantema polimorfo & Sí & Sí & Sí & Sí & Sí & Sí & Sí & Sí \\
\hline $\begin{array}{l}\text { Linfadenopatía cervical } \\
>1,5 \mathrm{~cm}\end{array}$ & No & No & No & No & No & No & No & No \\
\hline Síntomas respiratorios & No & No & Rinorrea, Tos & $\begin{array}{l}\text { Dificultad } \\
\text { Respiratoria }\end{array}$ & No & Rinorrea, Tos & No & Tos \\
\hline Tratamiento & $\begin{array}{l}\text { IGIV, AAS, } \\
\text { prednisona, } \\
\text { enoxaparina, } \\
\text { HCQ, } \\
\text { azitromicina }\end{array}$ & $\begin{array}{c}\text { IGIV, } \\
\text { AAS, MP, } \\
\text { enoxaparina, } \\
\text { HCQ, } \\
\text { azitromicina }\end{array}$ & $\begin{array}{l}\text { IGIV, AAS, } \\
\text { MP, HCQ }\end{array}$ & $\begin{array}{l}\text { IGIV (2 dosis), } \\
\text { AAS, MP, } \\
\text { enoxaparina, } \\
\text { ivermectina }\end{array}$ & $\begin{array}{l}\text { IGIV, AAS, MP, } \\
\text { enoxaparina }\end{array}$ & $\begin{array}{c}\text { IGIV, } \\
\text { AAS, MP, } \\
\text { ivermectina }\end{array}$ & $\begin{array}{l}\text { IGIV ( } 2 \text { dosis), AAS, MP } \\
\text { enoxaparina, ivermectina, } \\
\text { noradrenalina, dopamina, } \\
\text { levosimendan }\end{array}$ & $\begin{array}{l}\text { IGIV, AAS, MP, } \\
\text { enoxaparina }\end{array}$ \\
\hline Antibioticoterapia & Ceftriaxona & $\begin{array}{l}\text { Ceftriaxona } \\
\text { Metronidazol }\end{array}$ & Ceftriaxona & Ceftriaxona & $\begin{array}{l}\text { Ceftriaxona } \\
\text { Metronidazol }\end{array}$ & Ceftriaxona & Ceftriaxona & Ceftriaxona \\
\hline Evolución & Favorable & Favorable & Favorable & $\begin{array}{c}\text { Favorable } \\
\text { después de 2da } \\
\text { dosis de IGIV }\end{array}$ & Favorable & Favorable & $\begin{array}{c}\text { Shock, pasó a UCI. } \\
\text { Extubado y sin inotrópicos } \\
\text { a las } 48 \text { hrs de } 2 \text { da dosis } \\
\text { de IGIV }\end{array}$ & Favorable \\
\hline Estancia hospitalaria (días) & 9 & 9 & 13 & 14 & 10 & 11 & 14 & 10 \\
\hline
\end{tabular}

TE: tiempo de enfermedad, IGIV: inmunoglobulina intravenosa, AAS: ácido acetilsalicílico, MP: metilprednisolona, HCQ: hidroxicloroquina, UCI: unidad de cuidados intensivos, VM: ventilación mecánica 
Tabla 2. Hallazgos laboratoriales, microbiológicos y de imágenes en pacientes con MIS-C asociado a COVID-19

\begin{tabular}{|c|c|c|c|c|c|c|c|c|}
\hline Variable & Caso 1 & Caso 2 & Caso 3 & Caso 4 & Caso 5 & Caso 6 & Caso 7 & Caso 8 \\
\hline $\mathrm{Hb}(\mathrm{VR}: \geq 11,5 \mathrm{~g} / \mathrm{dL})$ & 10,1 & 11 & 9,9 & 10,2 & 9,6 & 11,2 & 11,3 & 11,4 \\
\hline Leucocitos (VR: 4,5 - 13,5 x10 $/ \mathrm{mm}^{3}$ ) & 14,39 & 14,70 & 26,96 & 16,45 & 47,86 & 17,12 & 13,9 & 4,87 \\
\hline Neutrófilos (VR: 1,5 - 8,0 x103/mm mm $^{3}$ & 12,52 & 12,64 & 15,96 & 24,56 & 43,07 & 13,69 & 9,31 & 3,70 \\
\hline Linfocitos (VR: $1,5-6,0 \times 10^{3} / \mathrm{mm}^{3}$ ) & 0,86 & 1,91 & 7,00 & 0,82 & 2,87 & 3,25 & 0,55 & 0,39 \\
\hline Plaquetas (VR: 150 - $350 \times 10^{3} / \mathrm{mm}^{3}$ ) & 53 & 90 & 460 & 69 & 108 & 240 & 122 & 53 \\
\hline PCR (VR: $\leq 0,5 \mathrm{mg} / \mathrm{dL})$ & 37,03 & 24,47 & 11,05 & 26,1 & 43 & 20,83 & 16,65 & 11,79 \\
\hline VSG (VR: $\leq 10 \mathrm{~mm} / \mathrm{h})$ & - & 60 & 54 & 43 & 67 & 54 & 50 & 18 \\
\hline Ferritina (VR: 7 - $140 \mathrm{ng} / \mathrm{mL}$ ) & 688 & 1382 & 607 & 710 & 557 & 216 & 495 & 909 \\
\hline Dímero D (VR: <0,5 mg/L) & 3,85 & 6,25 & 1,27 & 2,09 & 2,42 & 1,42 & 3,68 & 7,47 \\
\hline Fibrinógeno (VR: 160 - 369 mg/dL) & 693 & 630,4 & 607,8 & 505 & 751,9 & 661,8 & 710,9 & 342 \\
\hline TP (VR: 11,68 - 14,21 s) & 12,1 & 12,3 & 13,3 & 11,6 & 12,9 & 12,8 & 11,8 & 13,3 \\
\hline TTPA (VR: 27,12 - 44,21 s) & 59,2 & 40,7 & 41,2 & 36,9 & 40,7 & 48,5 & 47,6 & 36,5 \\
\hline DHL (VR: 230 - 460 U/L) & 367 & 703 & 589 & 517 & 350 & 652 & 513 & 918 \\
\hline TGP (VR: 0 - 39 U/L) & 34 & 139 & 74 & 77 & 140 & 24 & 24 & 102 \\
\hline TGO (VR: 0 - $47 \mathrm{U} / \mathrm{L}$ ) & 35 & 97 & 60 & 35 & 163 & 41 & 58 & 80 \\
\hline Urea (VR: 10 - 38 mg/dL) & 23 & 92 & 14 & 102 & 60 & 27 & 16 & 31 \\
\hline Creatinina (VR: 0,3 - 0,7 mg/dL) & 0,46 & 0,91 & 0,38 & 1,41 & 0,55 & 0,37 & 0,69 & 0,47 \\
\hline Sodio (VR: 135 - $148 \mathrm{mmol} / \mathrm{L}$ ) & 133 & 124 & 136 & 128 & 139 & 130 & 122 & 125 \\
\hline Albúmina (VR: 3,2 - 5,2 g/dL) & - & 3 & 3,1 & 2,4 & 2,8 & 2,3 & 2,8 & 2,4 \\
\hline Triglicéridos (VR: <150 mg/dL) & 216 & 363 & 168 & 158 & 154 & 162 & 80 & 246 \\
\hline CK-MB (VR: 0 - 24 U/L) & 10,5 & 12 & 16 & 19 & 11 & 17 & 19 & 42 \\
\hline RT-PCR COVID-19 & Negativo & Negativo & Positivo & Negativo & Negativo & Negativo & Negativo & Negativo \\
\hline IgM/IgG COVID-19 & $+/+$ & $+/+$ & $+/+$ & $+/+$ & $-1+$ & $+/+$ & $-1+$ & $-/+$ \\
\hline Hemocultivo & Negativo & Negativo & Negativo & Negativo & Negativo & Negativo & Negativo & Negativo \\
\hline Coprocultivo & Negativo & Negativo & Negativo & - & Negativo & - & - & - \\
\hline Radiografía de tórax & Normal & $\begin{array}{c}\text { Infiltrado } \\
\text { intersticial, } \\
\text { cardiomegalia }\end{array}$ & Normal & $\begin{array}{l}\text { Infiltrado } \\
\text { intersticial }\end{array}$ & $\begin{array}{l}\text { Infiltrado } \\
\text { intersticial, } \\
\text { cisuritis }\end{array}$ & Normal & Normal & Normal \\
\hline Ecografía abdominal & - & $\begin{array}{l}\text { Liquido libre } 200 \\
\text { cc en hemiabdo- } \\
\text { men inferior }\end{array}$ & - & - & $\begin{array}{c}\text { Proceso } \\
\text { inflamatorio } \\
\text { fosa iliaca } \\
\text { derecha }\end{array}$ & - & - & $\begin{array}{c}\text { Hepato- } \\
\text { esplenomegalia } \\
\text { leve }\end{array}$ \\
\hline Electrocardiograma & Normal & BIRD & Normal & Normal & Normal & Normal & Normal & Normal \\
\hline Ecocardiograma & Normal & $\begin{array}{l}\text { FSVI levemente } \\
\text { deprimida, } \\
\text { efusión pericár- } \\
\text { dica leve. }\end{array}$ & Normal & Normal & Normal & Normal & $\begin{array}{c}\text { Pericardio } \\
\text { hiperrefringente, } \\
\text { dilatación leve } \\
\text { del VI, insufi- } \\
\text { ciencia mitral } \\
\text { leve }\end{array}$ & Normal \\
\hline
\end{tabular}

Hb: hemoglobina, VR: valores de referencia, PCR: proteína C reactiva, VSG: velocidad de sedimentación globular, TP: tiempo de protrombina, TTPA: tiempo parcial de tromboplastina activada, DHL: lactato deshidrogenasa, TGP: alanina aminotransferasa, TGO: aspartato aminotransferasa, CK-MB: creatina quinasa-MB, COVID-19: enfermedad por coronavirus 2019, BIRD: bloqueo incompleto de rama derecha, FSVI: función sistólica ventricular izquierda, VI: ventrículo izquierdo.

20 de junio del 2020. La descripción detallada de hallazgos clínicos, laboratoriales, tratamiento y evolución se presentan en Tablas 1 y 2 .

La mediana de edad fue de 5,5 años, cinco eran varones, solo uno presentó como comorbilidad obesidad; a excepción del caso 5, todos tuvieron contacto con casos intradomiciliarios infectados por SARS-CoV-2.

La mediana del tiempo de enfermedad al momento de hospitalización fue de 4 días (rango: 4 a 7 días). Todos cursaron con fiebre, síntomas gastrointestinales, inyección conjuntival y exantema; cinco presentaron compromiso en cavidad oral y extremidades (Figura 1). La mediana de duración de la fiebre al momento del diagnóstico fue de 4,5 días (rango: 3 a 7 días). Cuatro presentaron criterios para EK completo y tres para EK incompleto. Cuatro cursaron con síntomas respiratorios, uno de ellos con distrés que requirió oxígeno suplementario (caso 4) (Tabla1).

Mayormente el diagnóstico fue al momento de la hospitalización. El caso 2 tuvo diagnóstico al ingreso de sepsis por apendicitis probable, el caso 6 por deshidratación e intoxicación alimentaria, en ambos el compromiso ocular y mucocutáneo apareció al tercer día de hospitalización completando 


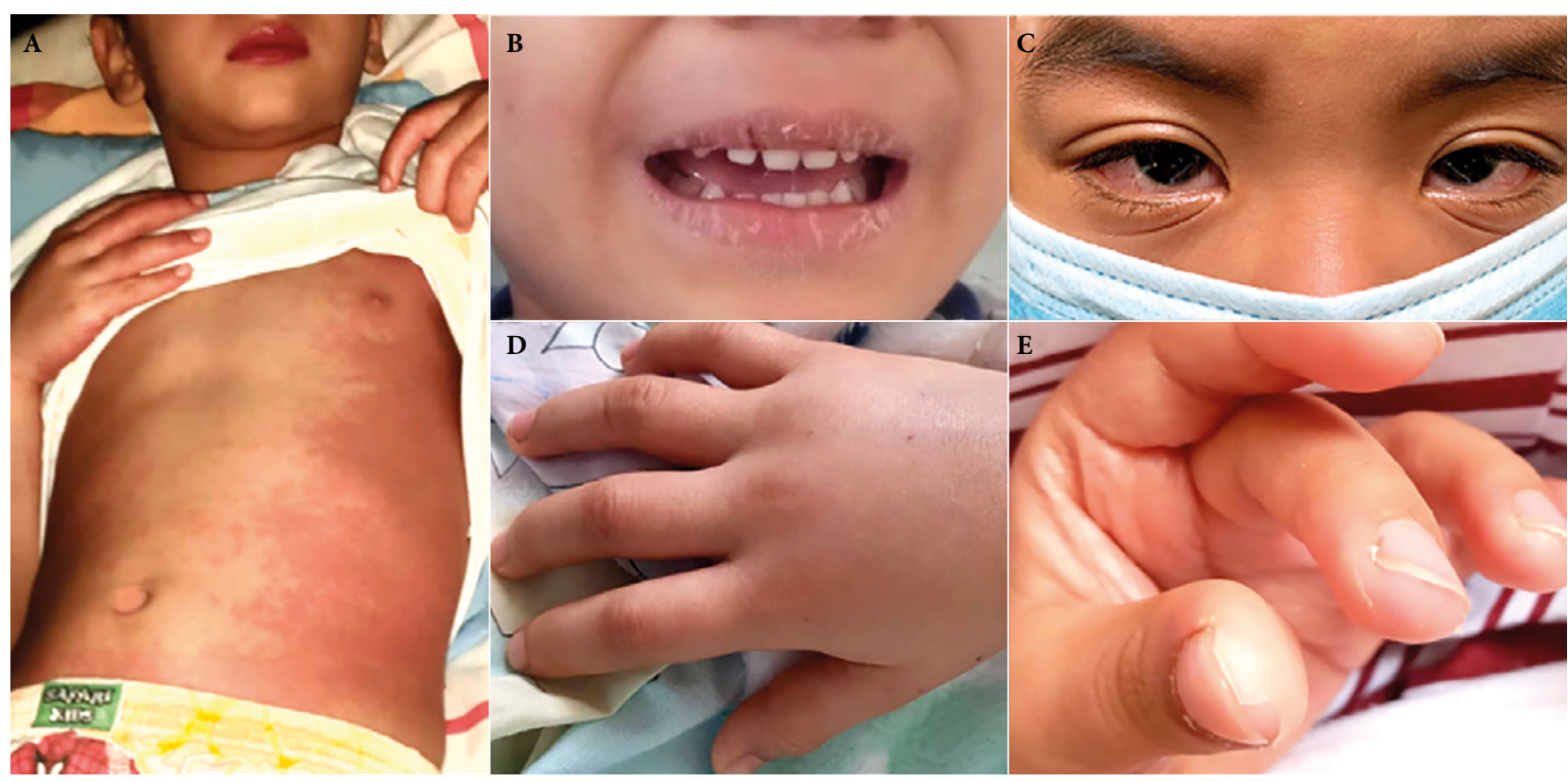

Figura 1. A. Rash maculopapular difuso, eritema y edema de manos, labios eritematosos (caso 1). B. Sequedad y fisura de labios (caso 4). C. Inyección conjuntival (caso 8). D. Edema en dorso de mano y dedos (caso 2). E. Descamación de pulpejos de dedos (caso 1). (Imágenes compartidas con autorización de los padres)

criterios para MIS-C. El caso 5 estuvo hospitalizado en otro nosocomio por apendicitis probable, al ingreso a nuestra institución se evidenció que cumplía criterios para MIS-C.

Antes de iniciar tratamiento, a todos se les realizó prueba molecular y detección de anticuerpos mediante prueba rápida. Todos presentaron anticuerpos para SARS-CoV-2: cinco tenían IgM e IgG, y tres tenían solo IgG; solamente el caso 3 tuvo prueba molecular positiva (Tabla 2). Las alteraciones en el hemograma fueron frecuentes: todos cursaron con anemia, siete presentaron leucocitosis y neutrofilia, cuatro linfopenia, seis trombocitopenia y uno trombocitosis. Todos presentaron proteína $\mathrm{C}$ reactiva, velocidad de sedimentación globular, ferritina y dímero D elevados. Siete tuvieron hiperfibrinogenemia, hipoalbuminemia e hipertrigliceridemia. Seis presentaron lactato deshidrogenasa elevadaehiponatremia.Cincopresentaronhipertransaminasemia $\mathrm{y}$ tres retención nitrogenada. Tres tuvieron tiempo parcial de tromboplastina activada prolongado. Solo uno presentó creatina quinasa-MB elevada. Cuatro pacientes cumplieron criterios laboratoriales para SAM (Tabla 3). Tres tenían radiografía torácica y ecografía abdominal patológica respectivamente, dos presentaron ecocardiograma patológico (Tabla 2).

Tabla 3. Riesgo de resistencia a inmunoglobulina intravenosa según puntaje de Kobayashi y criterios laboratoriales para Síndrome de Activación Macrofágica en pacientes con MIS-C asociado a COVID-19 ${ }^{(15-16)}$.

\begin{tabular}{|c|c|c|c|c|c|c|c|c|}
\hline Variable & Caso 1 & Caso 2 & Caso 3 & Caso 4 & Caso 5 & Caso 6 & Caso 7 & Caso 8 \\
\hline Kobayasahi $\geq 5$ & Sí & Sí & No & Sí & Sí & Sí & Sí & Sí \\
\hline$<12$ meses (1pto) & No & No & Sí & No & No & No & No & No \\
\hline PCR $\geq 10 \mathrm{mg} / \mathrm{dL}$ (1pto) & Sí (37) & Sí $(24,47)$ & Sí $(11,05)$ & Sí $(26,1)$ & Sí (43) & Sí $(20,83)$ & Sí $(16,65)$ & Sí $(11,79)$ \\
\hline Neutrófilos $\geq 80 \%$ (2ptos) & Sí (87\%) & Sí (86\%) & No $(59 \%)$ & Sí (89\%) & Sí (90\%) & Sí (80\%) & No $(67 \%)$ & No $(76 \%)$ \\
\hline Plaquetas $\leq 300 \times 10^{3} / \mathrm{mm}^{3}$ (1pto) & Sí (53) & Sí (90) & No $(460)$ & Sí (69) & Sí (108) & Sí (240) & Sí (122) & Sí (53) \\
\hline Sodio $\leq 133 \mathrm{mmol} / \mathrm{L}$ (2ptos) & No (133) & Sí (124) & No (136) & Sí (128) & No (139) & Sí (130) & Sí (122) & Sí (125) \\
\hline $\mathrm{TGO} \geq 100 \mathrm{U} / \mathrm{L}(2 \mathrm{ptos})$ & No (35) & No (97) & No $(60)$ & No (35) & Sí (140) & No $(41)$ & No $(58)$ & No $(80)$ \\
\hline $\begin{array}{l}\leq 4 \text { días de enfermedad al momento de } \\
\text { iniciar IGIV ( } 2 \text { ptos) }\end{array}$ & Sí & No & No & No & No & No & Sí & Sí \\
\hline SAM & Sí & Sí & No & Sí & No & No & No & Sí \\
\hline Ferritina $>684 \mathrm{ng} / \mathrm{ml}$ y 2 de los siguientes: & Sí (688) & Sí (1382) & No (589) & Sí (710) & No (557) & No (216) & No (495) & Sí (909) \\
\hline Plaquetas $\leq 181 \times 10^{3} / \mathrm{mm}^{3}$ & Sí (53) & Sí (90) & No $(460)$ & Sí (69) & Sí (108) & No (240) & Sí (122) & Sí (53) \\
\hline $\mathrm{TGO}>48 \mathrm{U} / \mathrm{L}$ & No (35) & Sí (97) & Sí (60) & No (35) & Sí (140) & No (41) & Sí (56) & Sí (70) \\
\hline Triglicéridos $\geq 156 \mathrm{mg} / \mathrm{dL}$ & Sí (216) & Sí (363) & Sí (168) & Sí (158) & No (154) & Sí (162) & No $(80)$ & Sí (246) \\
\hline Fibrinógeno $\leq 360 \mathrm{mg} / \mathrm{dL}$ & No (693) & No $(630,4)$ & No $(607,8)$ & No (505) & Sí $(751,9)$ & No $(661,8)$ & No $(710,9)$ & Sí (342) \\
\hline
\end{tabular}

SAM: Síndrome de Activación Macrofágica 
Como tratamiento inicial todos recibieron inmunoglobulina intravenosa (IGIV) a $2 \mathrm{gr} / \mathrm{kg} / \mathrm{dosis}$ (dosis máxima: $70 \mathrm{gr}$ ), ácido acetilsalicílico (AAS) (inicialmente a 50-80 mg/ $\mathrm{kg} /$ día y luego a $5 \mathrm{mg} / \mathrm{kg} /$ día tras 48 horas afebril), y corticoides (prednisona o metilprednisona) a $2 \mathrm{mg} / \mathrm{kg} /$ día por 5 días (luego disminución progresiva completando 2 semanas). La necesidad de corticoides se determinó mediante evaluación del riesgo de resistencia a la IGIV: score de Kobayashi $(\mathrm{SK}) \geq$ $5 \mathrm{y} / \mathrm{o}$ presencia de MAS y/o edad $\leq 12$ meses. Siete pacientes tenían un $\mathrm{SK} \geq 5$, cuatro cumplían criterios de MAS (Tabla 3), uno era menor de 12 meses, por lo que todos fueron tratados concomitantemente con corticoides. Los casos 4 y 7 requirieron segunda dosis de IGIV. Seis recibieron enoxaparina a dosis profiláctica debido a las alteraciones significativas en las pruebas de coagulación (dímero D: 4-15 veces su valor basal, hiperfibrinogenemia). Todos iniciaron antibioticoterapia empírica que fue suspendida tras cultivos negativos. Solo uno requirió vasopresores (Tabla 1 ).

En cuanto a la respuesta al tratamiento y evolución, el caso 4 persistió febril y con distrés 48 horas después de iniciada IGIV, por lo que se indicó segunda dosis con respuesta favorable a las veinticuatro horas. El caso 7 presentó shock refractario a fluidos e inició vasopresores, tras 36 horas no hubo mejoría clínica indicándose segunda dosis de IGIV, e ingresó a cuidados intensivos (UCI) requiriendo ventilación mecánica. El paciente fue extubado y se suspendieron los vasopresores 48 horas después de la segunda dosis de IGIV, permaneció 8 días en UCI. El resto de pacientes respondieron favorablemente al tratamiento inicial, la fiebre cedió 24-36 horas después de iniciar tratamiento, mientras que los síntomas gastrointestinales y mucocutáneos resolvieron máximo al cuarto día. Ningún paciente presentó efectos adversos al tratamiento recibido. Todos los pacientes fueron finalmente dados de alta, con indicación de continuar con AAS a $5 \mathrm{mg} / \mathrm{kg} /$ día por al menos 4 semanas y reducción progresiva de corticoides. La mediana de la estancia hospitalaria fue de 10,5 días (rango: 9 a 14 días).

\section{DISCUSIÓN}

Se desconoce la fisiopatología del MIS-C, se sugiere sería resultado de una respuesta inmune anormal al virus por similitudes con EK, SST y SAM ${ }^{(4,5)}$. El pico de casos se daría 2-4 semanas después del pico de casos de COVID-19 en la comunidad, coincidiendo con el momento de la inmunidad adquirida y sugiriendo que se presentaría como complicación posinfecciosa $^{(7,9-11)}$.

En investigaciones previas los casos de MIS-C se presentaron en un periodo de 10-54 días ${ }^{(3,7-11)}$. En este reporte, los 8 casos se presentaron en un periodo de 26 días. En el Perú, La Torre realizó un estudio en nuestra institución, encontrando 17 casos de EK en un periodo de 8 años ${ }^{(12)}$, Culqui et al. reportaron 42 casos de EK en 12 años en dos hospitales del seguro social de Lima ${ }^{(13)}$.
La edad promedio fue 5,1 años, pero el $75 \%$ tenía 5 años o más. Investigaciones previas han reportado edades medias entre 6,5 a 8 años ${ }^{(3,7-11)}$, lo cual difiere de EK que afecta principalmente a menores de 5 años ${ }^{(7,14)}$. En Lima, Culqui et al. encontraron que $50 \%$ de sus casos de EK eran menores de 2 años ${ }^{(13)}$. Se ha descrito preponderancia de raza negra e hispana en MIS-C ${ }^{(3,10,11)}$, en contraste la EK es más frecuente en asiáticos ${ }^{(14)}$, ninguno de nuestros pacientes tenía ascendencia asiática. Algunos reportes describen comorbilidades en 12 a $28 \%$ de casos, principalmente asma y obesidad ${ }^{(8,9,11)}$, uno de nuestros pacientes presentaba obesidad.

Respecto al cuadro clínico, coincidimos con lo descrito en estudios previos que reportan fiebre en $100 \%$ de casos, síntomas gastrointestinales en 80 a 100\%, grados variables de compromiso ocular y mucocutáneo en 30 a $80 \%$, y 42 a $82 \%$ de casos que cumplían criterios de EK completo ${ }^{(3,7-11)}$. Se observó que los síntomas gastrointestinales precedieron al compromiso dermatológico, hecho ya resaltado previamente en otros estudios ${ }^{(9,10)}$. Menos del 30\% de casos previamente reportados presentaron síntomas respiratorios y/o neurológicos ${ }^{(7,9-11)}$, lo cual difiere de nuestro estudio donde el $50 \%$ presentaron síntomas respiratorios y ninguno síntomas neurológicos. Como se describió, en dos de nuestros casos (casos 2 y 5) inicialmente se sospechó de apendicitis; Belhadjer et al. describieron también dos casos que incluso requirieron cirugía y resultaron ser adenitis mesentérica ${ }^{(8)}$.

Nuestros hallazgos laboratoriales coinciden con las investigaciones previas ${ }^{(3,7-11)}$, y es evidencia del marcado estado inflamatorio y compromiso multisistémico del MIS-C. Usualmente la EK cursa con anemia, leucocitosis, trombocitosis y marcadores inflamatorios elevados; en contraste, la presencia de linfopenia, trombocitopenia, compromiso renal, hepático y pruebas de coagulación alteradas son hallazgos menos frecuentes en $\mathrm{EK}{ }^{(14)}$, pero se han descrito ampliamente en MIS-C ${ }^{(3,7-11)}$. Solo un paciente presentó alteración de enzimas cardiacas, en estudios previos más del $50 \%$ de casos presentaron dicha alteración ${ }^{(7-11)}$. Se ha descrito SAM en menos del $2 \%$ de casos de $\mathrm{EK}^{(15)}$, Verdoni et al. lo reportaron en $50 \%$ de sus casos de MIS-C ${ }^{(7)}$, la mitad de nuestros pacientes tenían SAM.

Todos nuestros pacientes tuvieron serología positiva para SARS-CoV-2, el $100 \%$ fueron IgG positivo y el $62,5 \%$ eran también IgM positivo. Estudios previos reportan que 80 a $90 \%$ de sus pacientes tuvieron serología positiva: $50-90 \%$ a IgG, 6-30\% a IgG e IgM; además el 20 a 40\% tenían PCR positivo ${ }^{(7,9-11)}$. Estos hallazgos evidenciarían infección previa por SARS-CoV-2 en los pacientes que desarrollan MIS-C, lo que apoya una posible asociación temporal.

Estudios previos describen alteraciones en la radiografía de tórax en $50 \%$ de casos, principalmente efusión pleural ${ }^{(3,7,10)}$, en nuestra serie tres pacientes presentaron infiltrados intersticiales bilaterales. Solo dos presentaron ecocardiograma patológico, pero no aneurismas coronarios. Reportes previos describen alteraciones ecocardiográficas en $38-100 \%$ de pacientes (principalmente disfunción ventricular izquierda y efusión pericárdica), menos del $20 \%$ presentaron aneurismas coronarios ${ }^{(3,7-11)}$. 
Debido a las similitudes de este nuevo síndrome con EK, ha sido tendencia emplear el tratamiento estándar para EK que incluye principalmente IGIV y AAS, y corticoides en casos de alto riesgo de resistencia a la IGIV ${ }^{(3,7-11,14)}$. En reportes previos el 70$100 \%$ de casos recibieron IGIV, el 33-88\% corticoides ${ }^{(3,7-11)}$. El uso de AAS fue más variable; $75 \%$ de casos reportados por Riphagen et al. y $100 \%$ de casos de Verdoni et al, recibieron AAS a dosis antiinflamatorias (30-80 mg/kg/día) ${ }^{(3,7)}$. Estudios posteriores no reportan el uso de AAS ${ }^{(8,9)}$. Toubiana et al. reportan su uso en $100 \%$ de sus pacientes, pero solo a dosis antiagregantes (3-5mg/kg/día) ${ }^{(10)}$. Todos nuestros pacientes recibieron IGIV, corticoides y AAS. La necesidad de corticoide se determinó valorando el riesgo de resistencia a IGIV con la edad del paciente, el SK ${ }^{(16)}$, y la presencia de SAM ${ }^{(15)}$. Verdoni et al. reportaron que $70 \%$ de sus pacientes con MIS-C tuvieron alto riesgo de resistencia a IGIV ${ }^{(7)}$, en nuestro estudio el 100\% de casos lo presentaba. Esto explicaría la mayor necesidad de corticoides en MIS-C ${ }^{(3,7-11)}$, en contraste con EK donde generalmente menos del $20 \%$ de casos presentan resistencia a la IGIV y requieren corticoides ${ }^{(14,16)}$.

Solo dos de nuestros casos (25\%) requirieron segunda dosis de IGIV, Toubiana et al. reportaron necesidad de segunda dosis en el $24 \%$ de sus pacientes ${ }^{(10)}$. El $75 \%$ de nuestros pacientes recibieron anticoagulación profiláctica, Belhadjer et al. reportaron que el $65 \%$ de sus pacientes recibieron dosis terapéuticas de heparina ${ }^{\left({ }^{8}\right)}$, Cheung et al.describen el uso de enoxaparina profiláctica en el 59\% de sus pacientes ${ }^{(11)}$. Sin embargo, no existe aún evidencia para inicio de anticoagulación profiláctica en MIS-C.

Solo un paciente presentó miocarditis, shock, y requirió soporte ventilatorio, lo cual difiere marcadamente de estudios previos donde el $50-100 \%$ de casos presentaron miocarditis y shock, e ingresaron a UCI y el 40-80\% requirieron soporte ventilatorio $^{(3,7-10)}$. La estancia hospitalaria fue más prolongada que las reportadas en estudios anteriores (estancias hospitalarias medias entre 7,1-10 días, rango 3-18 días) ${ }^{(8,10,11)}$. Como ya se mencionó, la mayoría de nuestros pacientes respondieron con éxito al tratamiento inicial; la estancia hospitalaria prolongada se debió principalmente a demoras en la realización de los estudios ecocardiográficos por razones logísticas. No reportamos ningún deceso, solo dos estudios previos reportaron un fallecido respectivamente ${ }^{(3,9)}$.

En conclusión, el MIS-C sería una forma grave del COVID-19 en niños. No está clara aún la relación etiológica, pero su presentación coincidente en el tiempo con la pandemia y la preponderancia de serología para SARS-CoV-2 (principalmente IgG) en los pacientes afectados, avalarían una posible asociación. Como hemos descrito, a pesar de sus similitudes clínicas y laboratoriales con otras patologías (principalmente EK), se han ido estableciendo algunas características que lo diferencian como la edad de presentación, raza, síntomas gastrointestinales preponderantes, mayor compromiso multisistémico, marcadores inflamatorios marcadamente alterados, mayor frecuencia de miocarditis, shock y SAM y mayor necesidad de uso de corticoides. Sin embargo, se ha observado que su espectro clínico es amplio y varía entre un síndrome febril persistente con evidencia de inflamación, una presentación similar a EK, SAM y una forma más severa con shock, miocarditis y falla multiorgánica. Debido a su potencial gravedad es recomendable que, frente a todo niño con fiebre, síntomas gastrointestinales y mucocutáneos, asociado a exposición al SARS-CoV-2, debe investigarse compromiso multisistémico para diagnóstico precoz y tratamiento oportuno que mejore el pronóstico.

Contribución de los autores: LEDV, MKZA, HNP, RBM, CFCT, MCDLP han participado en la concepción y diseño del artículo. LEDV, MKZA, HNP, RBM, CLHM, SCSM, JLCH, FAP y RARG han participado en la interpretación de datos y revisión crítica del artículo. Todos los autores participaron en la recolección de datos, redacción y aprobación de la versión final.

Financiamiento: Autofinanciado.

Conflictos de interés: Los autores declaran no tener ningún conflicto de interés en ninguna de las etapas del desarrollo del artículo.

Consideraciones éticas: Todos los padres dieron su consentimiento para el reporte de los casos.

\section{REFERENCIAS BIBLIOGRÁFICAS}

1. Ludvigsson JF. Systematic review of COVID-19 in children shows milder cases and a better prognosis than adults. Acta Paediatr. 2020;109(6):108895. doi: 10.1111/apa.15270.

2. Jones VG, Mills M, Suarez D, Hogan CA, Yeh D, Segal JB, et al. COVID-19 and Kawasaki Disease: Novel Virus and Novel Case. Hosp Pediatr. 2020;10(6):537-40. doi: 10.1542/hpeds.2020-0123.

3. Riphagen S, Gomez X, Gonzalez-Martinez C, Wilkinson N, Theocharis P. Hyperinflammatory shock in children during COVID-19 pandemic. Lancet. 2020;395(10237):1607-8. doi: 10.1016/S0140-6736(20)31094-1.

4. Royal College of Paediatrics and Child Health Guidance - Paediatric multisystem inflammatory syndrome temporally associated with COVID-19 (PIMS) [Internet]. RCPCH. [citado 25 de junio de 2020]. Disponible en: https://www.rcpch.ac.uk/resources/guidance-paediatric-multisystem-inflammatory-syndrome-temporally-associated-covid-19-pims.
5. World Health Organization. Multisystem inflammatory syndrome in children and adolescents temporally related to COVID-19 [Internet]. [citado $25 \mathrm{de}$ junio de 2020]. Disponible en: https://www.who.int/news-room/commentaries/detail/multisystem-inflammatory-syndrome-in-children-and-adolescents-with-covid-19.

6. Health Alert Network (HAN): Multisystem Inflammatory Syndrome in Children (MIS-C) Associated with Coronavirus Disease 2019 (COVID-19). [citado 25 de junio de 2020]. Disponible en: https://emergency.cdc.gov/ han/2020/han00432.asp.

7. Verdoni L, Mazza A, Gervasoni A, Martelli L, Ruggeri M, Ciuffreda M, et al. An outbreak of severe Kawasaki-like disease at the Italian epicentre of the SARS-CoV-2 epidemic: an observational cohort study. Lancet. 2020;395(10239):1771-8. doi: 10.1016/S0140-6736(20)31103-X. 
8. Belhadjer Z, Méot M, Bajolle F, Khraiche D, Legendre A, Abakka S, et al. Acute heart failure in multisystem inflammatory syndrome in children (MIS-C) in the context of global SARS-CoV-2 pandemic. Circulation. 2020. doi: 10.1161/CIRCULATIONAHA.120.048360.

9. Whittaker E, Bamford A, Kenny J, Kaforou M, Jones CE, Shah P, et al. Clinical Characteristics of 58 Children With a Pediatric Inflammatory Multisystem Syndrome Temporally Associated With SARS-CoV-2. JAMA. 2020; e2010369. doi: 10.1001/jama.2020.10369.

10. Toubiana J, Poirault C, Corsia A, Bajolle F, Fourgeaud J, Angoulvant F, et al. Kawasaki-like multisystem inflammatory syndrome in children during the covid-19 pandemic in Paris, France: prospective observational study. BMJ. 2020; 369. doi: 10.1136/bmj.m2094.

11. Cheung EW, Zachariah P, Gorelik M, Boneparth A, Kernie SG, Orange JS, et al. Multisystem Inflammatory Syndrome Related to COVID-19 in Previously Healthy Children and Adolescents in New York City. JAMA. 2020. doi: 10.1001 /jama.2020.10374.

12. La Torre E. Hallazgos clínicos en paciente con Enfermedad de Kawasaki en el Instituto Nacional de Salud del Niño durante el periodo 2000-2007. [tesis]. Lima: Facultad de Medicina, Universidad Nacional Mayor de San Marcos; 2009. Biblioteca San Fernando, Lima, Perú.

13. Culqui K, Ávila C, Moisés C, Carril M, Rodríguez J, Quispe M, et al. Enfermedad de Kawasaki: comportamiento clínico y complicaciones cardiovasculares en niños hospitalizados en dos Centros Hospitalarios de IV nivel, 2000-2012. Rev Peru Pediatr. 2013; 66 (4): 215-222.

14. McCrindle BW, Rowley AH, Newburger JW, Burns JC, Bolger AF, Gewitz $\mathrm{M}$, et al. Diagnosis, Treatment, and Long-Term Management of Kawasaki Disease: A Scientific Statement for Health Professionals From the American Heart Association. Circulation. 2017; 135(17): e927-99. doi: 10.1161/CIR.0000000000000484.

15. Wang W, Gong F, Zhu W, Fu S, Zhang Q. Macrophage activation syndrome in Kawasaki Disease: More common than we thought? Semin Arthritis Rheum. 2015;44(4):405-10. doi: 10.1016/j.semarthrit.2014.07.007.

16. Kobayashi T, Inoue $Y$, Takeuchi K, Okada Y, Tamura K, Tomomasa T, et al. Prediction of intravenous immunoglobulin unresponsiveness in patients with Kawasaki disease. Circulation. 2006;113(22):2606-12. doi: 10.1161/ CIRCULATIONAHA.105.592865. 